\title{
El nuevo paradigma agrosocial, futuro del nuevo campesinado emergente
}

\author{
Neus Monllor \\ La Caseta de la Coma de Burg, Lleida, España: Email: neus@laccb.cat
}

Resumen: En este artículo se ha estudiado la realidad del relevo generacional en la agricultura. Los resultados demuestran cómo las prácticas y actitudes de los jóvenes varían en función de su origen familiar: los que han nacido en un entorno agrario (agricultores tradicionales) son más propensos a seguir las prácticas agrícolas dentro de un paradigma convencional, mientras que los jóvenes que provienen de familias no agrarias (agricultores nuevos) muestran características distintas más cercanas a un nuevo paradigma. La muestra de jóvenes confirma la existencia de un grupo que pone en práctica un Nuevo paradigma agrosocial donde los temas agrícolas y sociales toman un peso significativo. Este grupo de personas arraigadas a la tierra, que busca su propia autonomía y que se mantienen optimistas sobre el futuro es El Nuevo campesinado.

Palabras clave: estudio comparativo, joven agricultor, nuevo campesinado, nuevo paradigma agrosocial, relevo generacional.

\section{The new agrosocial paradigm, the future of the new emerging peasantry}

\begin{abstract}
This paper has explored the reality of generational renewal in agriculture. The results verify how the practices and attitudes of young people vary depending on the family origin: those who were born in an agrarian environment (continuers) are more likely to continue agricultural practices within a conventional paradigm, whereas young people who come from non-agrarian families (newcomers) show distinctive features that relate to a new paradigm. The sample of young people confirms the existence of a group that puts into practice a New agrosocial paradigm where farming and social issues take a significant weight. This group of people rooted to the land, searching their own autonomy and being optimistic about the future can be called "New peasantry".

Key words: comparative analysis, young farmer, new peasantry, new agrosocial paradigm, generational renewal.
\end{abstract}

\section{O novo paradigma agrosocial, futuro dos novos camponeses emergentes}

Resumo: Neste trabalho, estuda-se a realidade da mudança geracional na agricultura. Os resultados mostram como as práticas e atitudes dos jovens, variam de acordo com o seu ambiente familiar: os que nascem em um ambiente agrícola (agricultores tradicionais) são mais propensos a seguir as práticas agrícolas dentro do paradigma convencional, enquanto os jovens que vêm de famílias não-agríco- 
las (agricultores novos) mostram características diferentes mais perto de um novo paradigma. A amostra de jovens confirma a existência de um grupo que implementa um novo paradigma agrosocial onde as questões agrícolas e social assumem significativa. Este grupo de pessoas enraizadas na terra, em busca de sua própria autonomia e permanecem otimistas sobre o futuro é o campesinato novo.

Palavras-chave: estudo comparativo, jovem agricultor, novo paradigma campesinato agrosocial nova geração.

$$
* * *
$$

\section{Nuevo siglo, nuevos paradigmas}

El siglo XXI ha abierto sus puertas con una gran lista de interrogantes sobre los modelos sociales y económicos de esta nueva etapa. Los grandes avances tecnológicos de los países industrializados han transformado radicalmente las relaciones de las personas con su entorno. Muchos de estos cambios, más allá de aportar bienestar y calidad de vida, han interrumpido dinámicas saludables ancestrales, necesarias hoy en día para la supervivencia de nuestro planeta.

En el mundo rural el cambio vino dado por una potente industrialización de las actividades agrarias, una expulsión masiva de sus habitantes y un desprecio generalizado por las connotaciones culturales que el mundo rural irradiaba. En pocos años las actividades tradicionales de los pueblos se vieron reducidas, muchas de las cuales desaparecieron dando paso a la modernidad, la velocidad y la pérdida de identidad. Buena parte de los agricultores fueron expulsados de sus tierras por no alcanzar los estándares de productividad exigidos por el mercado, dejando un camino estéril para las nuevas generaciones.

El paradigma de la industrialización consiguió, en parte, su objetivo de desvincular las actividades agrarias de las personas y de su entorno. Por suerte, no todos los agricultores se convirtieron en productores o desaparecieron, sino que una buena parte resistió y reivindicó su condición campesina (Ploeg, 2008). La industrialización agresiva está siendo respondida por todas aquellas personas que no siguieron la rueda del desarrollo a toda costa, y por una nueva generación que confía en el compromiso social de las personas que trabajan la tierra y de las que consumen sus alimentos. Éste es el cambio de paradigma que está alimentando un nuevo campesinado emergente en infinitos rincones del mundo.

\section{El Nuevo paradigma agrosocial}

Para entender con más precisión esta nueva realidad económica y social del mundo agrario en territorios postindustriales, se llevó a cabo una investigación doctoral que muestra la realidad del proceso de incorporación a la actividad agraria en dos áreas de estudio: el sudoeste de la provin- 
cia de Ontario en Canadá y la provincia de Girona en España (Monllor, 2011). Con el fin de determinar el perfil de las nuevas generaciones se formuló una proposición hipotética donde se distinguen dos grupos: los que relevan la actividad agraria familiar (agricultores tradicionales) y los que entran de nuevo al sector agrario (agricultores nuevos). Se decidió poner a prueba esta cuestión mediante el establecimiento de grados de diferencia observando las prácticas y las actitudes de los jóvenes agricultores. Para poder establecer niveles comparativos de análisis fue necesario crear un marco teórico a partir del cual se pudieran establecer diferencias entre los grupos de estudio.

Metodológicamente se partió de la idea de la OCDE sobre el Nuevo Paradigma Rural (OCDE, 2006) con el fin de conceptualizar una teoría útil para testarla con la realidad. La OCDE plantea un cambio de paradigma en el mundo rural basado en la importancia del lugar frente a la importancia de los sectores, en la relevancia de las inversiones ante las subvenciones y en la articulación del territorio a partir de una nueva gobernanza rural. Según Crosta (2006) estos tres elementos son los que resumen una nueva manera de entender el mundo rural y las políticas que le afectan, creando un escenario nuevo lleno de incertidumbres y nuevas oportunidades. Esta idea de cambio de paradigma fue contrastada con otras cuatro teorías, que posteriormente formarían la base del Nuevo paradigma agrosocial.

La primera fue la de Bryden (2000), que sintetiza algunos de los rasgos que definen la nueva ruralidad del siglo XXI en: (1) esfuerzo en para reforzar las economías rurales, (2) intento de reestructuración de la agricultura, (3) fortalecimiento de la infraestructura de transporte y telecomunicaciones, (4) asistencia comercial y empresarial, (5) desarrollo de los recursos humanos mediante la formación profesional, (6) formación empresarial, (7 ) iniciativas school-to-work, (8) creación de capacidades locales, (9) creación de productos basados en la identidad local, (10) instrumentos financieros nuevos o adaptados, y (11) nuevas maneras de acercar los servicios públicos a las zonas rurales. Casi todos estos componentes se pueden identificar con el Nuevo paradigma rural de la OCDE donde se valora prioritariamente la escala local y las capacidades endógenas del territorio, y donde se prioriza la creación de vínculos de comunicación hacia la escala global. Al mismo tiempo, el autor refuerza el valor de la persona emprendedora como elemento axial de la nueva economía rural. Para Bryden, en el marco de un nuevo paradigma son primordiales la formación y la capacitación de las personas que han de formar parte de su propio desarrollo. La formación, los nuevos instrumentos financieros y las relaciones más flexibles con la administración fortalecen la capacidad tanto del campesinado como del resto de colectivos que conviven en los espacios rurales.

En segundo lugar, Acosta (2008) define en un contexto latinoamericano la nueva ruralidad a partir de ocho elementos: (1) territorio, (2) sostenibilidad, (3) capital social, (4) perspectiva de género, (5) contrarreforma agraria , (6) refuerzo de las instituciones en el marco de la 
reforma estatal, (7) participación democrática y (8) desarrollo comunitario y autogestión. Ella misma explica que ninguno de los componentes son nuevos aisladamente, lo que realmente aporta novedad es que estos se amalgaman felizmente para dar como resultado un paradigma de lo rural que se reivindica a sí mismo como ampliado, incluyente y multidimensional. Comparte con el paradigma de la OCDE el enfoque territorial, el fomento de la participación y el reforzamiento de la gobernanza local. Pero la autora añade algunos matices en cuanto a los temas sociales como la mirada de género, el intento de reformar estructuras agrarias enquistadas y el fortalecimiento del capital social como medida de compromiso social.

En tercer lugar, se estudiaron los diez principios fundadores de la agricultura campesina según Bove y Dufour (2003): (1) repartir los volúmenes de producción para permitir acceder al oficio de agricultor y de vivir un número máximo de personas, (2) ser solidario con los campesinos de las otras regiones del Mundo, (3) respetar la naturaleza, (4) valorar los abundantes recursos y ahorrar los recursos escasos, (5) transparencia en los actos de compra, de producción y de venta de los productos agrícolas, (6) asegurar la bondad gustativa y sanitaria de los productos, (7) alcanzar una autonomía máxima en el funcionamiento de las explotaciones agrarias, (8) buscar la afinidad con otros actores del mundo rural, (9) mantenimiento de la diversidad de las poblaciones animales de crianza y de las variedades vegetales cultivadas, (10) pensar siempre a largo plazo y de una manera global ligada al desarrollo sostenible. Estos principios fundadores de la agricultura campesina están muy cerca de un cambio de paradigma en el mundo rural. Los autores hacen un esfuerzo para reivindicar el papel del sector agrario arraigado a la tierra, de las relaciones de confianza entre la misma agricultura y entre la sociedad, así como de la ralentización de los procesos de producción para que sean más respetuosos con las generaciones futuras y con otros lugares del planeta.

Finalmente, se analizó el enfoque que Ploeg (2008) detalla a través de los nueve rasgos diferenciales de la condición campesina: (1) coproducción, (2) base de recursos autocontrolada, (3) ordenación de las relaciones con los mercados que aseguren autonomía, (4) supervivencia, (5) fortalecimiento de la base de recursos, (6) reducción de la dependencia, (7) lucha por la autonomía, (8) pluriactividad y (9) modelos de cooperación. Estos nueve elementos que presenta el autor ponen el campesinado como actor de la obra que representan. El desarrollo rural pasa porque el sector agrario se desate de los lazos que el sistema capitalista le ha atado y vuelva a escribir su propia historia. La manera de hacer campesina que describe el autor aporta una mirada más allá del Nuevo paradigma rural planteado por la OCDE.

Estos cuatro autores identifican diferentes elementos que forman parte de un modelo teórico basado en evidencias desde la praxis y que metodológicamente han habido de descomponer, para posteriormente poder analizar la realidad y volverla a rehacer. El Nuevo paradigma agrosocial que se explica en este artículo parte del análisis de los autores citados, de la 
literatura complementaria y de la propia experiencia personal. El resultado es la definición de 8 componentes: (1) escala local, (2) diversidad, (3) medio ambiente, (4) cooperación, (5) innovación, (6) autonomía, (7) compromiso social y (8) ralentización (Figura 1).

Figura 1: el Nuevo paradigma agrosocial.

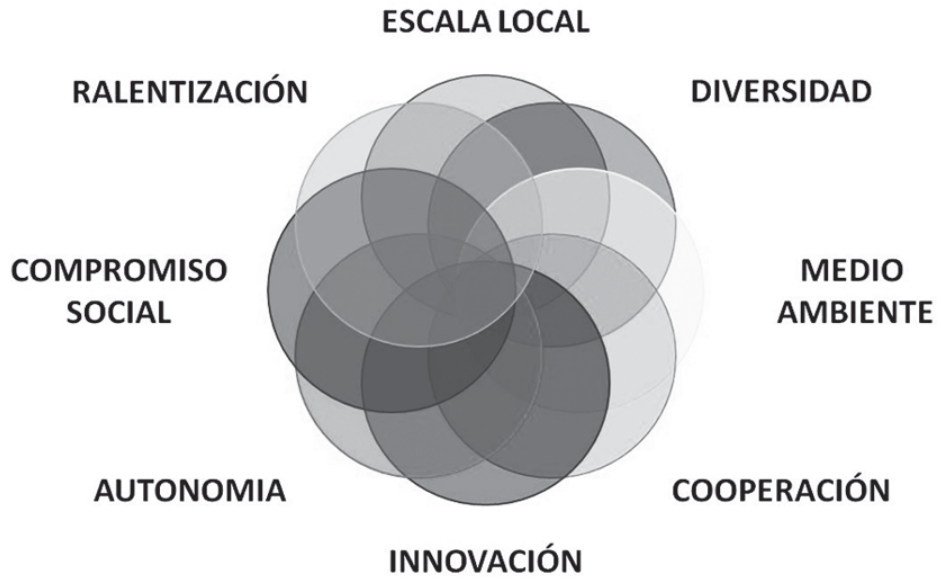

Escala local. Uno de los componentes básicos para encaminar un nuevo modelo de desarrollo rural hacia un escenario más equitativo es la revalorización de la escala local. La capacidad de comunicación a un nivel global ha desvirtuado el punto de vista local haciendo del mundo un espacio único de actuación. Esta nueva dimensión evoca a volver a valorar el lugar como un espacio auténtico, lleno de identidad y generador de oportunidades (Castells, 1998). Cuando se prioriza la escala local, los recursos próximos se convierten en una fuente única de desarrollo endógeno. En este marco es tan importante reconocer que existe un conocimiento y una sabiduría autóctona, como tomar en consideración que el fomento de los servicios a las pequeñas comunidades rurales es un signo de vitalidad y de apoyo al sector agrario del futuro (Bryden, 2000).

Uno de los elementos revolucionarios de los últimos años por parte del campesinado es la recuperación de las cadenas cortas de comercialización. Frente al anonimato de los productos de los centros comerciales una parte del campesinado reivindica el comercio directo y de proximidad. El número de iniciativas en todo el mundo donde se valoran las personas que producen alimentos se han multiplicado en la última década (Pérez-Vitoria, 2010). 
Dar importancia a la escala local es una manera directa de poner en marcha medidas de desarrollo rural desde el mismo territorio, ya que practicar una agricultura y una ganadería conscientes de la localidad aporta un valor añadido de primer orden. Las dinámicas que se generan trabajando a una escala cercana son las que en buena parte definen el nuevo paradigma. El territorio cobra sentido más allá de los sectores que lo componen y se entiende como un todo que funciona de manera orgánica.

Diversidad. La diversidad, ya sea en cultivos, en actividades, en actores sociales o en canales de comercialización es vital para caminar hacia un cambio de paradigma. Apostar por una amplia variedad de estrategias forma parte de entender la realidad desde la complejidad. Esta idea es antagónica al que el modelo industrial ha promulgado con los monocultivos, la concentración en la producción de materias primas o los contratos de integración ganadera, donde los agricultores asumen un mero rol de producción de materias primas para la agroindustria donde la diversificación no tiene sentido. De hecho, muchas de las actividades que implican un proceso de diversificación no son nuevas, sino más bien recuperadas de las antiguas prácticas agrarias previas a la Revolución verde. Es por este motivo que muchas veces la definición de diversificación se explica a través de la implantación de actividades alternativas (Bowler, 1996), alternativas al sistema convencional de la agricultura productivista dominante.

Desde un punto de vista sociológico también hay que tener en cuenta la diversidad de actores que conforman la nueva ruralidad. Los grupos tradicionales donde la agricultura local era la dominante y donde los roles estaban muy bien marcados se va segregando. El nuevo escenario acepta y adopta como oportunidad nuevos grupos sociales que dinamicen el espacio rural donde se insertan. En este caso los jóvenes recién llegados son un ejemplo claro. Años atrás eran tratados de hippies o liberales, pero hoy ya se empieza a entender que su integración es un factor de futuro y que hay que abrir los ojos para ver más allá de los grupos sociales que tradicionalmente han marcado el territorio (Moyano, 2000).

Medio Ambiente. Entendido como la relación entre el ser humano y la madre tierra, el medio ambiente es un elemento imprescindible de un modelo de desarrollo rural acuerde con el territorio y con el nuevo paradigma agrosocial. Las relaciones históricas que han caracterizado las actividades agrarias y forestales con su entorno han sido, en muchos de los casos, de integración y respeto.

El equilibrio, en el sentido de capacidad de asimilar los cambios, ha estado presente en las relaciones que han mantenido la agricultura y el medio ambiente hasta hace casi cincuenta años. A partir de los años sesenta, el proceso de industrialización, rápido, voraz y poco solidario, desequilibra unas relaciones tradicionalmente saludables. El afán productivista acelera el proceso de mecanización, de utilización de productos químicos y de intensificación de buena parte de las explotaciones agrarias de la época. En consecuencia el ítem principal de producir más para el mercado y al menor 
coste, se olvida del medio ambiente (Gómez Mendoza, 2001: 114; Ploeg, 2010: 100). Toledo et al. (1999) sitúan la problemática ambiental como el reto más grande y urgente de la ciencia contemporánea y argumentan los efectos nocivos de la agricultura industrial sobre el medio y los recursos naturales.

Por su parte, cuando se habla de agricultura y medio ambiente aparece la agricultura ecológica como el sistema de producción agraria que más se acerca a las dinámicas naturales de los ecosistemas. Sus inicios se remontan a los años setenta donde, en paralelo al proceso de industrialización de la agricultura moderna, se va configurando una manera alternativa de hacer agricultura. La evolución de la producción agraria ecológica es diferente en todos los países del mundo, pero es a partir de los años noventa que cobra más importancia. Cada vez el consumo ecológico es más elevado y por tanto la producción también crece y camina hacia su reconocimiento social.

Cooperación. La cooperación se puede dar entre personas, entidades o territorios. Las dimensiones son múltiples como también lo son las fórmulas para hacerlo. Es una estrategia que fomenta relaciones de confianza, de asociación y de fidelidad. Es a través de la cooperación que nace el capital social, definido como el conjunto de normas de confianza, valores, actitudes y redes entre personas e instituciones en un territorio o sociedad (Moyano, 2008). Estas normas facilitan acciones colectivas y de cooperación en tanto que diferentes actores sociales trabajan por la realización de un proyecto común (Tisenkopfs et al., 2010).

Des de un punto de vista agrario, se coopera cuando se interactúan con otros actores ya sean locales o de otros territorios. Lo que se comparte es un objetivo común, que enriquece el proceso de desarrollo y aporta elementos innovadores a las tradicionales formas de hacer (Ploeg y Marsden, 2010). Es por este motivo que la integración de agentes dispares bajo los mismos parámetros conforma un componente de riqueza y de competitividad desde la autonomía de cada uno de los miembros que ponen en marcha la actividad conjunta.

Bajo un nuevo paradigma rural la competitividad se logra a partir de la cooperación entre los diferentes agentes locales. Compite el territorio para dar salida a la gente que lo habita. Basada en la teoría de los sistemas locales territoriales se entiende que el espacio rural a desarrollar es una entidad autónoma y rica en recursos propios cultivados históricamente (Dematteis, 2002). Esta se convierte en un nodo activo de una red global que conecta con el mundo y con las novedades que continúan nutriendo, pero siempre desde una base arraigada de capacidades locales y endógenas. La teoría de las redes da un valor extraordinario a la cooperación entre los diferentes agentes del territorio.

Innovación. La innovación es necesaria para repensar y volver a formular muchos de los procesos viciados que no funcionan en determina- 
dos ambientes agrarios y/o rurales. La innovación también permite salir del ámbito local para ir a buscar nuevas maneras de hacer que complementen los conocimientos tradicionales o de escala local. Este componente aporta elementos de novedad totalmente imprescindibles para crear economías dinámicas. El valor local es vital, pero no es suficiente para mirar hacia el futuro (Capello, 1996).

Durante las últimas décadas la innovación tecnológica y la agricultura industrial han sido los argumentos dominantes para producir alimentos. Las explotaciones agrarias familiares tuvieron que adaptarse o abandonar. El campesinado dejaba de innovar porque eran los grandes centros de investigación y las casas comerciales las que proponían y creaban, las que venían y luego se iban. Se produjo una sustitución de la tecnología agraria de naturaleza artesanal a una de naturaleza industrial (Sevilla Guzmán, 2006: 12).

El nuevo paradigma se acerca más al concepto de innovación de los jóvenes que ponen en marcha estrategias liberadoras de las cargas que la agroindustria impone cada vez con más fuerza a quienes trabajan la tierra. Entiende la innovación en su vertiente más humana y cercana a la gente y a su lucha por ser cada día más autónomos e independientes, más cooperativos que competitivos, más ligados al territorio que al capital. En este contexto se valora la invención de soluciones nuevas a partir de las necesidades existentes y los recursos disponibles.

La innovación que defiende el nuevo escenario rural es la que cree en el valor añadido de la producción de los alimentos de la tierra, la que crea nuevas maneras de acercarse al consumidor o que inventa relaciones de confianza para hacer viable la empresa agraria. Es la búsqueda continúa para experimentar desde la propia imaginación.

Autonomía. El Nuevo paradigma agrosocial apunta tendencias hacia estadios más autónomos en contrapartida al antiguo paradigma de la modernización. Desde un punto de vista estructural es totalmente conocida la pérdida de autonomía y de decisión del campesinado a raíz de integrarse en los circuitos agroindustriales (Aldomà, 2009: 124). La pseudoseguridad que desprenden algunas de las empresas que controlan la cadena, ha hecho que la mayor parte del tejido agrario haya perdido las riendas de sus operaciones cotidianas como la elección y reproducción de semillas, la alimentación del ganado o la comercialización de los propios productos.

Esta dependencia no es admitida por todo el conjunto del campesinado. De hecho en muchos casos se utilizan estrategias de distanciamiento y de independencia respecto a los abusos del modelo industrial. Ploeg (1994) llama a este fenómeno resistencia social, donde se refleja que una buena parte del campesinado ha respondido a la uniformidad de la visión general de las políticas y prácticas que dominaron el viejo paradigma de la modernización a ultranza. Las explotaciones agrarias son autónomas en la medida que reducen su dependencia de factores externos que limitan la toma de decisiones. El campesinado que desarrolla estrategias de reforzamiento 
desde su base de recursos reafirma su autonomía frente a la dependencia de los recursos externos (Ploeg, 2008; Bové y Dufour, 2003).

Algunas estrategias como la pluriactividad o la venta directa están encaminadas a hacer de las explotaciones agrarias espacios de producción más autónomos. Poner el precio al producto que los campesinos producen es un símbolo claro de autonomía y en muchos casos una oportunidad de hacer más viable la actividad agraria (Seccombe, 2007).

Compromiso social. El compromiso social se identifica como uno de los ocho componentes claves en tanto que se entiende que la actividad agraria va más allá de la mera producción de alimentos. El campesinado que se acerca al nuevo paradigma es cercano al territorio, a sus raíces y sobre todo a su gente. El compromiso social hace referencia a una mirada que traspasa el objetivo económico de producir alimentos y de vivir en un entorno rural, para valorar otros aspectos sociales y ambientales (Riechman, 2003; Rooij, 2006).

Las estrategias que responden a este componente desde las explotaciones agrarias están focalizadas a entender que hay que crear modelos que vayan más allá de lo que es únicamente económico, que hay que pensar en las generaciones futuras a la vez de implementar nuevas prácticas agrarias y que, en la medida que se pueda, hay que crear situaciones simbióticas donde todas las partes salen ganando. Las empresas agrarias que asumen este compromiso suelen alcanzar roles más proactivos en la organización social de las redes de las que forman parte.

El compromiso social también se puede medir en función de la relación que se establece en el territorio donde se vive. Es importante que una nueva generación de campesinos y campesinas fomenten el empleo local a raíz de la creación de actividades agrarias integradas en las redes de la comunidad rural. En este sentido es necesario entender que no toda la agricultura genera los mismos puestos de trabajo. Estudios recientes muestran como la producción agraria ecológica fomenta la creación de puestos de trabajo locales (Maynard y Green, 2006), mientras que la agricultura mecanizada tiene cada vez menos capacidad de absorber mano de obra y generar empleo (Etxezarreta, 2006: 91).

También se visualiza el compromiso social del campesinado cuando se piensa más allá del cultivo del año, cuando se incorporan las generaciones futuras en las acciones presentes. En este sentido la proliferación de los Organismos Genéticamente Modificados (OGM) que ha hecho caso omiso al Principio de precaución, es un tema donde la agricultura tiene mucho que decir. Ikerd (2000: 5) argumenta que no puede haber una agricultura futura sin una armonía ecológica, una viabilidad económica y una responsabilidad social hacia las generaciones venideras.

Ralentización. La ralentización es un componente que reclama el nuevo paradigma en su concepción. Hay que reducir la velocidad desde un 
punto de vista amplio para disfrutar del proceso y de poder adoptar estrategias de flexibilidad. Las nuevas prácticas y la introducción de novedades requieren una manera de hacer poco a poco para coger conciencia de la novedad y para reducir el riesgo de la incertidumbre que provocan los procesos de aprendizaje (Ploeg, 2000). Frente a la desmesurada velocidad de algunos de los cambios que el proceso de modernización agraria ha inculcado a los sistemas agrarios, se valora un ritmo continuo pero sin prisas, de adopción de nuevas técnicas agrarias.

También se ralentiza cuando se siguen los ciclos de la naturaleza. La modernización y la industrialización de muchos de los procesos agrarios han desvirtuado la relación de la producción de alimentos con los tempos que corresponden desde un punto de vista tradicional y han intensificado en muchos casos los métodos de producción. Por tanto, la ralentización también hace referencia a los procesos de desintensificación, a adecuar en buena medida los límites de los factores de producción y a hacer de la actividad agraria una práctica más humana, dentro de una escala manejable y flexible. En este sentido, también incluye la reducción de la dependencia de las energías fósiles (Pérez-Vitoria, 2010) y introduce el criterio de autonomía con respecto al uso de energías no renovables. Cuanto más autosuficiente sea una explotación agraria más futuro se le augura.

El componente de la ralentización también entiende que no es necesario ser grande para ser competitivo (Schumacher, 1983 [1973]) y que se pueden aumentar los márgenes netos de una actividad agraria a partir de diferentes estrategias. Además hay que tener en cuenta que las empresas agrarias de dimensiones reducidas llevan a cabo muchas otras funciones más allá de la propiamente productivas. Gómez Mendoza (2001: 120) también reafirma esta visión, donde vincula la relación de las explotaciones agrarias pequeñas con la gestión del territorio y del paisaje.

\section{El Índice del Nuevo paradigma agrosocial}

A partir de la estructura teórica del Nuevo paradigma agrosocial se creó una nueva herramienta de análisis: El Índice del Nuevo paradigma agrosocial. El Índice fue construido en dos partes bien diferenciadas. La primera está relacionada con las prácticas que se llevan a cabo en el día a día de la explotación agraria, y la segunda, con la actitud de los jóvenes agricultores en relación con el presente y el futuro de la agricultura. El Índice mide el lugar aproximado donde el joven se sitúa entre un viejo y un nuevo paradigma (el Indice está construido en una escala de 0 a 10, siendo el 10 la puntuación máxima y más cerca del nuevo paradigma agrosocial).

Para la primera parte, la que hace referencia a las prácticas agrarias, se tuvieron en cuenta dos elementos para medir cada componente, por tanto, un total de 16 unidades de análisis que han permitido construir la parte del Índice que hace referencia (tabla 1). 
Tabla 1: Elementos para medir las prácticas agrarias.

\begin{tabular}{|c|c|c|}
\hline COMPONENTE & $\begin{array}{l}\text { PRÁCTICAS: } \\
\text { ELEMENTO } 1\end{array}$ & $\begin{array}{l}\text { PRACTICAS: } \\
\text { ELEMENTO 2 }\end{array}$ \\
\hline ESCALA LOCAL & El ámbito geográfico de la venta directa & $\begin{array}{l}\text { La identificación local del } \\
\text { producto }\end{array}$ \\
\hline DIVERSIDAD & La diversificación de la actividad económica & $\begin{array}{l}\text { La diversidad de los canales de } \\
\text { comercialización }\end{array}$ \\
\hline MEDIO AMBIENTE & $\begin{array}{l}\text { Las mejoras ambientales en la explotación } \\
\text { agraria }\end{array}$ & La producción agraria ecológica \\
\hline COOPERACIÓN & La cooperación con otros agricultores & $\begin{array}{c}\text { La cooperación con agentes no } \\
\text { agrarios }\end{array}$ \\
\hline INNOVACIÓN & La incorporación de prácticas innovadora & $\begin{array}{l}\text { La redacción de un plan de } \\
\text { empresa }\end{array}$ \\
\hline AUTONOMIA & El sentirse atrapado por alguna cosa & El poner el precio al producto \\
\hline COMPROMÍSO SOCIAL & La vinculación al tejido asociativo & $\begin{array}{l}\text { El ser miembro activo en alguna } \\
\text { asociación }\end{array}$ \\
\hline RALENTIZACIÓN & $\begin{array}{l}\text { La afectación del incremento del precio del } \\
\text { gasoil }\end{array}$ & $\begin{array}{l}\text { El grado de inversión en el } \\
\text { punto de partida }\end{array}$ \\
\hline
\end{tabular}

Para la segunda parte, la que hace referencia a las actitudes de los jóvenes hacia un nuevo paradigma agrosocial, se han tenido en cuenta tres elementos para cada componente, con un total de 24 unidades de análisis (tabla 2).

\section{Tabla 2: Elementos para medir las actitudes hacia un nuevo paradigma agrosocial.}

\begin{tabular}{|c|c|c|c|}
\hline COMPONENTE & $\begin{array}{c}\text { ACTITUD: } \\
\text { ELEMENTO } 1\end{array}$ & $\begin{array}{c}\text { ACTITUD: } \\
\text { ELEMENTO } 2\end{array}$ & $\begin{array}{c}\text { ACTITUD: } \\
\text { ELEMENTO } 3\end{array}$ \\
\hline ESCALA LOCAL & $\begin{array}{l}\text { La escala local como estrategia de } \\
\text { futuro }\end{array}$ & $\begin{array}{l}\text { La recuperación de } \\
\text { variedades y razas } \\
\text { tradicionales }\end{array}$ & $\begin{array}{l}\text { La moda de comprar y } \\
\text { comer productos locales }\end{array}$ \\
\hline DIVERSIDAD & $\begin{array}{c}\text { El suporte público a las } \\
\text { explotaciones agrarias para que se } \\
\text { diversifiquen }\end{array}$ & $\begin{array}{l}\text { La multifuncionalidad } \\
\text { de la actividad agraria }\end{array}$ & $\begin{array}{l}\text { La diversidad de las } \\
\text { personas agricultoras }\end{array}$ \\
\hline MEDIO AMBIENTE & $\begin{array}{l}\text { El suporte público al fomento de } \\
\text { prácticas ambientales en las } \\
\text { explotaciones agrarias }\end{array}$ & $\begin{array}{c}\text { Las prácticas agrarias } \\
\text { modernas tienen en } \\
\text { cuenta el medio } \\
\text { ambiente }\end{array}$ & $\begin{array}{l}\text { La calidad de los } \\
\text { alimentos producidos } \\
\text { ecológicamente }\end{array}$ \\
\hline COOPERACIÓN & $\begin{array}{c}\text { La cooperación con entidades no } \\
\text { agrarias }\end{array}$ & $\begin{array}{l}\text { La cooperación entre } \\
\text { los agricultores }\end{array}$ & $\begin{array}{c}\text { La cooperación entre } \\
\text { los diferentes agentes } \\
\text { que han de intervenir en } \\
\text { politica rural }\end{array}$ \\
\hline INNOVACIÓN & $\begin{array}{l}\text { La experimentación con nuevas } \\
\text { idees }\end{array}$ & $\begin{array}{l}\text { La innovación de los } \\
\text { agricultores desde sus } \\
\text { recursos propios }\end{array}$ & $\begin{array}{c}\text { La importancia de la } \\
\text { tecnologia para ser } \\
\text { competitivo }\end{array}$ \\
\hline AUTONOMIA & $\begin{array}{c}\text { El control de la explotación } \\
\text { agraria }\end{array}$ & $\begin{array}{c}\text { La dependencia de la } \\
\text { tecnología agraria } \\
\text { moderna }\end{array}$ & $\begin{array}{c}\text { El papel de los } \\
\text { ayuntamientos en el } \\
\text { fomento de la } \\
\text { agricultura }\end{array}$ \\
\hline $\begin{array}{l}\text { COMPROMISO } \\
\text { SOCIAL }\end{array}$ & $\begin{array}{l}\text { La generación de puestos de } \\
\text { trabajo en áreas rurales }\end{array}$ & La utilización de OGM & $\begin{array}{l}\text { La implicación de los } \\
\text { agricultores en el } \\
\text { mantenimiento de las } \\
\text { áreas rurales }\end{array}$ \\
\hline RALENTIZACIÓN & $\begin{array}{l}\text { La intensificación de la } \\
\text { producción para obtener } \\
\text { beneficios }\end{array}$ & $\begin{array}{l}\text { La necesidad de ser } \\
\text { grande para ser } \\
\text { competitivo }\end{array}$ & $\begin{array}{l}\text { La introducción de } \\
\text { nuevas prácticas } \\
\text { agrarias } \\
\text { progresivamente }\end{array}$ \\
\hline
\end{tabular}


Las siguientes figuras muestran algunos de los resultados del análisis. En las dos primeras se exponen los valores del Índice en referencia a las prácticas agrarias. Se muestran los diferentes valores en función del origen familiar de las personas que se incorporan (figura 2), así como en función del origen familiar y geográfico (figura 3).

\section{Fig. 2: Valores del Índice de prácticas en función del origen familiar.}

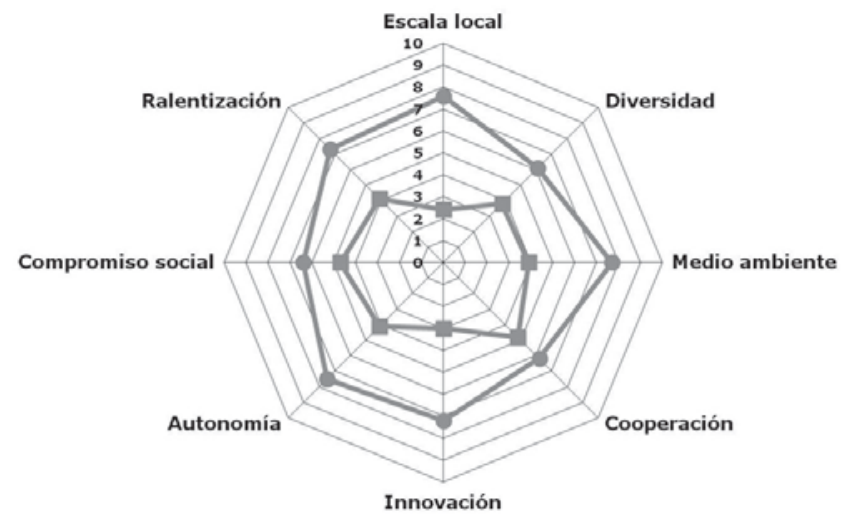

-Agricultores tradicionales $\quad$-Agricultores nuevos

Fig. 3: Valores del Índice de prácticas en función del origen familiar y geográfico.

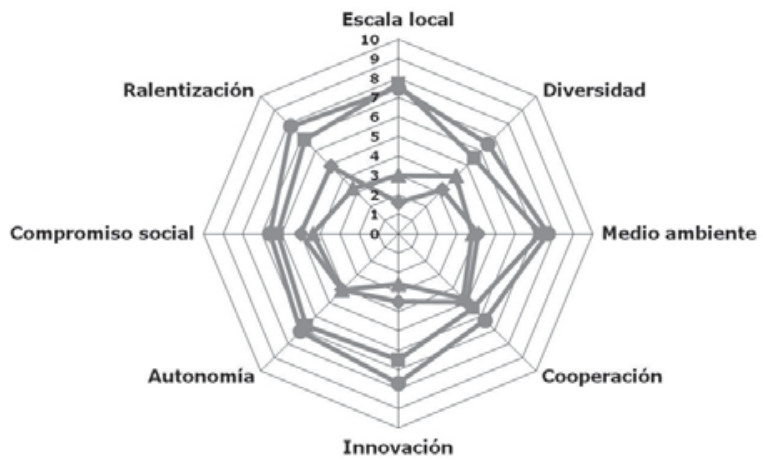

$\leftarrow$ Agricultores tradicionales Ontario 
Las prácticas son un reflejo de la realidad agraria en la vida cotidiana de las personas que trabajan la tierra. La figura 2 muestra las diferencias entre los agricultores tradicionales y los nuevos para cada uno de los ocho componentes del Nuevo paradigma agrosocial. El resultado es una posición muy diferente respecto al cambio de paradigma, y en consecuencia que la forma de actuar de las personas jóvenes se basa en dos modelos agrarios diferentes.

Por ejemplo, mientras los agricultores tradicionales han perdido la escala local vendiendo sus productos a la agroindustria, los nuevos han adoptado estrategias de viabilidad de sus empresas a partir de la venda directa y de la relación personal con las personas consumidoras. Otra práctica que los separa enormemente es el tipo de producción que llevan a cabo. La gran mayoría de los tradicionales continúan el modelo productivista y convencional que han heredado de sus padres, en cambio los nuevos están convencidos de que la única vía de cultivar alimentos y criar ganado es a través de la producción ecológica. En este sentido también es interesante remarcar la dimensión física y económica de las actividades que gestionan los jóvenes. En general, los tradicionales están al mando de grandes empresas capitalizadas, especializadas y enfocadas a la productividad, mientras que los nuevos optan por dimensiones pequeñas que les permitan ser autónomos en su gestión sin entrar en una gran dependencia con los bancos, las multinacionales o la administración pública.

Si se comparan los resultados del análisis entre las dos áreas de estudio, la principal observación es que los agricultores son más similares en función de su origen familiar que de su ubicación geográfica. No encontramos diferencias significativas entre las prácticas agrarias de los jóvenes en Ontario y en Girona, hecho que nos permite pensar en que las dinámicas de los jóvenes en función de su origen familiar son similares en distintos sitios y lugares.

Las figuras 4 y 5 muestran los valores en referencia a las actitudes. La figura 4 compara los valores entre los jóvenes en función de su origen familiar, y la figura 5 en función de su origen familiar y geográfico.

Fig. 4:Valores del Índice de actitudes en función del origen familiar.

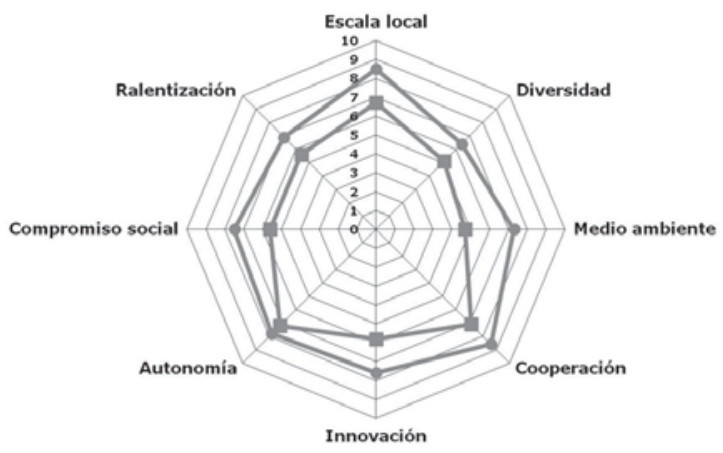

-Agricultores tradicionales

$\rightarrow$ Agricultores nuevos 


\section{Fig. 5: Valores del Índice de actitudes en función del origen familiar y geográfico.}

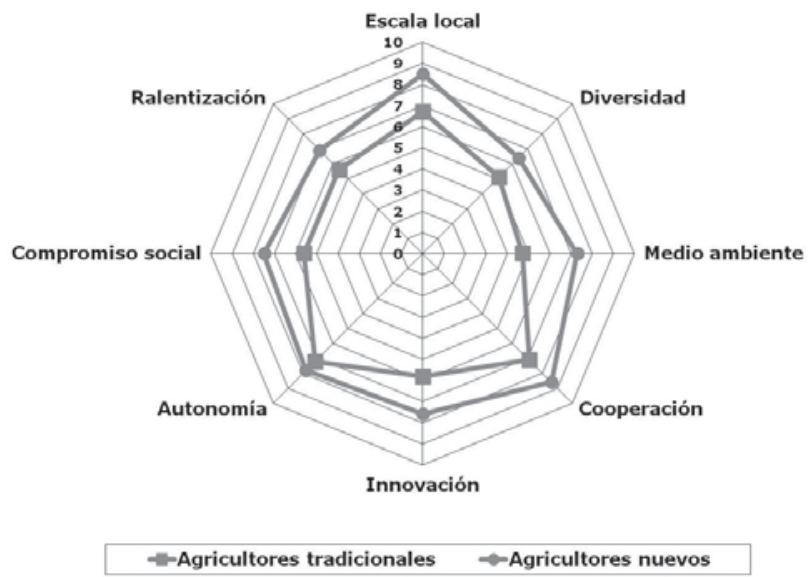

Las actitudes se refieren a la manera de pensar que tienen los jóvenes sobre el futuro de la agricultura y en la visión que expresan del mundo para comprender su realidad. La Figura 4 muestra cómo tanto los agricultores tradicionales como los nuevos están abiertos, en diferentes grados, a las nuevas ideas. Eso significa que la manera en que los agricultores están pensando en el futuro de la agricultura incluye los componentes del Nuevo paradigma agrosocial, y muestra como algunos de los tradicionales estaría dispuestos a introducir cambios en sus prácticas, que de momento se sitúan mucho más cerca del paradigma antiguo.

Por ejemplo, hay una visión compartida de que los agricultores tendrán que reorientar algunas de sus estrategias para producir alimentos para la población local. Tanto un grupo como el otro visualizan que el futuro del sector agrario pasa por recuperar la escala local en la comercialización de los alimentos que producen. También comparten la visión y el deseo de ser más autónomos respecto a los bancos, las grandes empresas, los productos químicos, etc. La gran mayoría de los tradicionales son conscientes del poco margen de maniobra que tienen, pero no se ven capaces de introducir cambios en sus prácticas. Los dos grupos también han demostrado la necesidad de cooperar entre ellos, aunque la mayoría cree que es difícil llevarlo a cabo.

El análisis también muestra diferencias de actitud en los dos grupos, en algunas cuestiones clave como el uso regular de los transgénicos o la importancia de la agricultura ecológica. Este tipo de elementos son los que separan los puntos de vista de los agricultores tradicionales y los nuevos. Mientras que para los nuevos la agricultura ecológica es la "única manera 
de hacer agricultura”, para la mayoría de los tradicionales es sólo una moda o una oportunidad de encontrar un mercado nuevo para sus productos.

La figura 5 muestra las diferencias entre los agricultores en relación a su origen geográfico, donde se observa para los cuatro grupos que la tendencia es a ser más abiertos hacia el nuevo paradigma que en sus prácticas. Este resultado permite ser optimista respecto al futuro de las nuevas generaciones. Los jóvenes que están relevando la actividad de sus padres se encuentran atrapados en un modelo agroindustrial que les da pocas opciones de cambio, pero de donde estarían motivados a salir; y los recién llegados se instalan en empresas nuevas que ya llevan el sello impreso del Nuevo paradigma agrosocial.

\section{El nuevo campesinado emergente}

Los resultados muestran como está naciendo un nuevo grupo de personas jóvenes que rompen con la tendencia agroindustrial de la mayor parte de empresas agrarias de los países occidentales. El análisis ha demostrado que el punto de partida en función del origen familiar incide directamente en la tipología de explotación agraria que se pone en marcha. Los que relevan a la familia continúan el modelo agroindustrial de crecimiento, especialización y productividad heredado de sus padres. Pocos son los que han invertido el camino y han optado por otro tipo de agricultura. En cambio, los agricultores nuevos se incorporan a la actividad agraria con una mirada renovada y más cercana a un nuevo paradigma.

Se constatan dos modelos agrarios diferentes en función del origen familiar del joven que se incorpora. En relación a las prácticas agrarias se evidencia una posición continuista por parte de los agricultores tradicionales, mientras que los nuevos arriesgan en poner en marcha nuevas estrategias de producción, comercialización y distribución. En cambio, el estudio de las actitudes muestra como los dos grupos se acercan al Nuevo paradigma agrosocial. Este hecho nos plantea una cuestión nueva en relación a la manera como los agricultores familiares ven y entienden el futuro del sector agrario.

Una de las principales aportaciones de esta investigación es la visualización de un grupo de agricultores y agricultoras que se encuentran cerca del Nuevo paradigma agrosocial. En su mayoría son nuevos, pero algunos de los tradicionales también están mostrando tendencias que los incluyen en a nueva ola emergente. A este grupo se le ha llamado Nuevo campesinado, siguiendo el marco teórico de The New Peasantry (Ploeg, 2008). Los nuevos campesinos están arraigados a la localidad, creen en la diversificación, promueven prácticas respetuosas con el medio ambiente, cooperan para avanzar en su causa común, introducen tecnologías apropiadas y de escala humana, luchan por su propia autonomía, tienen en cuenta las generaciones futuras y tratan de reducir la intensificación respecto a las prácticas agrícolas convencionales. Este Nuevo Campesinado, formado tanto 
por agricultores tradicionales como por nuevos, está dibujando el futuro de una renovada agricultura. Corroboran este resultado varios informes, libros y artículos que han aparecido en los últimos años que hablan de este grupo emergente como una solución clara y real a las continuas preguntas sobre la sostenibilidad y la incoherencia cada vez mayor del modelo agrícola imperante (Maynard y Green, 2006; Aurélie, 2007; Mailfert, 2007; Binimelis et al 2008; Ploeg, 2008; Webb, 2009; Gillespie y Johnson, 2010; Euskadiko Gazteriaren Kontseilua, 2010; Niewolny y Lillard, 2010; PérezVitoria, 2010).

En este sentido también se ha constatado que cada vez cobran más importancia los temas agrarios y sociales, tanto en las áreas urbanas como rurales. El estudio de los jóvenes, de sus prácticas y de sus actitudes nos permite afirmar que estamos asistiendo a un cambio de paradigma en términos de agrosociabilidad. Los ejemplos son cada vez más visibles, y van desde la organización de las cooperativas de consumo locales, al fortalecimiento de los mercados ecológicos o al papel visible de la mujer en el campo. El Nuevo paradigma agrosocial abre la puerta a que una renovada generación de campesinos y campesinas alimenten a sus gentes, labrando la tan preciada soberanía alimentaria.

Esta nueva realidad social muestra el surgimiento de una energía vital renovada para el cultivo de una nueva agricultura. Aunque Hervieu (1997:52) nos recuerda que al final del siglo pasado los campesinos se habían convertido en productores, Ploeg (2008) añade que, al mismo tiempo, el campesinado vuelve a aparecer precisamente como respuesta al antiguo paradigma marcado por la industrialización de la agricultura. La coexistencia de los dos modelos en el mismo espacio y tiempo crea "un campo de batalla complejo en el que diferentes intereses, perspectivas y proyectos tienen que competir” (Ploeg, 2008:151). En este sentido es interesar remarcar el papel estratégico de las personas que consumen alimentos, entendiendo que el poder de compra es infinito y que cada moneda dedicada al Nuevo campesinado forma parte del cambio. El surgimiento y revitalización de las actividades agrarias estratégicas para el territorio están totalmente vinculados al compromiso que adquieren los consumidores y las consumidoras con el nuevo modelo agrario.

Este compromiso es global, porque el modelo de consumo y producción de alimentos está totalmente unido a la calidad del entorno y a su revitalización futura, entendiendo que no todas las prácticas agrícolas promueven el desarrollo sostenible de un territorio. Las actividades llevadas a cabo por el Nuevo campesinado, entre otras cosas, fomentan el capital social, mantienen los ámbitos rurales y urbanos conectados, apuestan por la calidad óptima de los alimentos promoviendo así la salud de las personas, vuelven a habitan zonas abandonadas, crean nuevas relaciones sociales, proponen formas alternativas de organizarse y colaborar, tienen en mente las futuras generaciones y se esfuerzan por mantener la tan preciada multifuncionalidad de los espacios agrarios. 
Todos estos atributos son los que definen el desarrollo rural, que ha sido estudiado y revisado tanto por la literatura des de los años ochenta (Etxezarreta, 1987; Comisión Europea, 1988; Hervieu, 1997; Bryden, 2000; Ploeg et al. 2000; Viladomiu, 2003; Monllor et al. 2005; OCDE, 2006; O’Connor et al. 2006). Por lo tanto, las contribuciones económicas, sociales y ambientales del Nuevo campesinado deben ser tomadas seriamente en consideración para reactivar y renovar las áreas rurales. Las políticas públicas deben entender el Nuevo paradigma agrosocial como un modelo que proporciona calidad de vida, que conserva los recursos naturales y que hace que las áreas rurales sean económicamente mucho más viables.

El Nuevo campesinado emergente es una realidad internacional que permite pensar en positivo el futuro de las áreas rurales. La mayor parte son agricultores nuevos, pero también encontramos agricultores tradicionales que están modificando sus pautas y que están apostando por su territorio a partir de reinventar una nueva agricultura campesina. Encontramos personas con discursos optimistas, con una energía focalizada en la calidad y el trato personal y con un amor a la tierra que las mantiene vivas y activas.

En conclusión, lejos de prever la desaparición del campesinado, podemos afirmar un resurgimiento global que permite ser optimistas en términos de soberanía alimentaria. Las actividades del campo se están volviendo a valorar, y ser campesino ya no es visto con desprecio, sino con mucha honra. Las nuevas generaciones que acceden al sector agrario por vocación propia y las que desde la tradición se renuevan, tienen en sus manos el cambio hacia un modelo agrario mucho más justo, orgánico y solidario. 


\section{Bibliografía}

Acosta, I.L. (2008), “The paradigm of new rurality as the axis of public policies. What can we expect?”, en Revista electrónica Zacatecana sobre Población y Sociedad, 32.

Alberdi Collantes, J. (2005), “Jóvenes agricultores, perspectivas, planes de dinamización y dificultades de instalación en el País Vasco”, en Papeles de Geografía, 41-42, pp. 5-28.

Aldomà, I. (2009), Atles de la nova ruralitat. Fundació del Món Rural, Lleida.

Aurélie, A. (2007), La Vía Campesina. La globalización y el poder del campesinado. Editorial popular, Madrid.

Binimelis, R.; Bosch, M. i Herrero, A. (2008), A sol i serena. dones, món rural i pagesia. Institut Català de les Dones, Generalitat de Catalunya, Barcelona.

Bove, J. i Dufour, F. (2003), La llavor del futur. L'agricultura explicada als ciutadans. Pagès Editors, Lleida.

Bowler, I. (1985), Agriculture under the Common Agricultural Policy, a geography. Manchester University Press, Manchester.

Bryden, J. (2000), “Is there a New Rural Policy in OCDE countries?”, en International Conference on Rural Communities and Identities in the Global Millennium. Canada, 1-4 de mayo de 2000, Nanaimo.

Capello, R. (1996), "Industrial enterprises and economic space, the network paradigm”, en European Planning Studies, 4(4), pp. 485-498.

Castells, M. (1998), La Era de la Información. Alianza, Madrid.

Comissió Europea (1988), El futuro del mundo rural, comunicació de la Comisió al Consell i al Parlament. Ministerio de Agricultura, Pesca y Alimentación, Madrid.

Comissió Europea (1999), Estratègia Territorial Europea. Hacia un desarrolo equilibrado y sostenible de la UE. Comissió Europeu, Luxemburg.

Crosta, N. (2006), "Reinventing rural policy”, en Policy brief of the OCDE, octubre de 2006.

Dematteis, G. (2002), “De las regiones-área a las regiones-red. Formas emergentes de gobernabilidad regional”, en Subirats, J. (Coord.), Redes, territorios y gobierno. Diputació de Barcelona, pp. 163-175, Barcelona. 
Etxezarreta, M. (1987), El desenvolupament rural integrat. Barcelona, Diputació de Barcelona, Quaderns Rurals, Barcelona.

Ídem (coord.) (2006), La agricultura española en la era de la globalización. Ministerio de Agricultura, Pesca y Alimentación, Madrid.

Euskadiko Gazteriaren Kontseilua (2010), “Jóvenes Baserritarras. Situación socioeconómica de la juventud agroganadera en la CAPV”, en Hizpideak, 4, pp. 1-36.

Fennell, R. (1981), "Farm succesion in the European Community”, en Sociologia Ruralis, 21(1), pp. 19-41.

Gillespie Jr, G.W. i Johnson, S. (2010), "Success in farm start-up in the Northeastern United States”, en Journal of Agriculture, Food Systems and Community Development, 1.

Gómez Benito, C. i González, J.J. (2002), “Familia y explotación en la transformación de la agricultura española”, en Gómez Benito, C. i González, J.J. (Coord.), Agricultura y sociedad en el cambio de siglo. Madrid, McGraw Hill i UNED, pp. 427-450.

Gómez Mendoza, J. (2001), “Las nuevas funciones socioeconómicas y medioambientales de les espacios rurales”, en García Pascual, F. (coord.), El mundo rural en la era de la globalización, incertidumbres y potencialidades. Ministerio de Agricultura, Pesca y Alimentación, pp. 111-148, Madrid.

Hervieu, B. (1995), Hacia un nuevo sistema rural. Serie Estudios del Ministerio de Agricultura, Pesca y Alimentación, Madrid.

Langreo, A. (2000), "Innovaciones y desarrollo rural, nuevas iniciativas de empleo y juventud”, en Juventud Rural, Revista de Estudios de Juventud, 48, pp. 73-81.

Lasley, P. (2005), “All in the family, the decision to take up farming”, en Agricultural Outlook Forum 2005. Arlington, 24 de febrer de 2005.

Maynard, R. i Green, M. (2006), Organic works. Providing more jobs through organic farming and local food supply. Soil Association, Bristol.

Monllor, N. (2011), Explorant la jove pagesia, camins, pràctiques i actituds en el marc d'un nou paradigma agrosocial. Tesi doctoral, Universitat de Girona.

Monllor, N.; Roca, A.; Ribas, A. i Salamaña, I. (2005), Proposta i aplicació d'un model de contracte territorial al Pla de l'Estany. Girona, Departament d'Agricultura, Ramaderia i Pesca i Càtedra de Pensament Territorial de la Universitat de Girona. 
Moyano, E. (2000), "Procesos de cambio en la sociedad rural española. Pluralidad de intereses en una nueva estrutura de oportunidades”, en Papers, 61, pp. 191-200.

Ídem (2008), “Capital social, gobernanza y desarrollo en zonas rurales”, en Foro Internacional de Cooperación y Desarrollo Rural. 8-10 de junio de $2008,<$ v.

OCDE (Organització per a la Cooperació i el Desenvolupament Econòmics) (2006), New Rural Paradigm, policies and governance. OCDE, Bruselas.

O’Connor, D. et al. (Eds.), Driving Rural Development, Policy and Practice in Seven EU Countries. Royal van Gorcum, Assen, Països Baixos.

Perez-Vitoria, S. (2010), El retorno de los campesinos. Una oportunidad para nuestra supervivencia. Icaria, Barcelona.

Ploeg, J.D. van der (2008), The new peasantries. Struggles for autonomy and sustainability in an era of empire and globalization. Earthscan, Londres.

Ídem (2010), “The food crisis, industrialized farming and the imperial regime”, en Journal of Agrarian Change, 10(1), pp. 98-106.

Ploeg, J.D. van der i Long, A. (Eds.) (1994), Born from within, Practice and perspectives of endogenous rural development. Royal van Gorcum, Assen, Països Baixos.

Ploeg, J.D. van der i Marsden, T. (Eds.) (2010), Unfolding Webs. The dynamics of regional rural development. Royal van Gorcum, Assen, Països Baixos.

Regidor, J.G. (Coord.) (2008), Desarrollo rural sostenible, un nuevo desafío. Ministerio de Medio Ambiente, Rural y Marino, Madrid.

Riechmann, J. (2003), Cuidar la T(t)ierra. Políticas agrarias y alimentarias sostenibles para entrar en el siglo XXI. Icaria, Barcelona.

Rooij, S. de (2006), “Territorial cooperative networks, new social carriers for endogenous rural development”, en Haverkort, B. i Reijntjes, C. (Eds.), Moving worldviews, resahping ciencies, policies and practices for endogenous sustanaible development. COMPAS series on Worldviews and Sciencies, 4, pp. 237-253. ETC/COMPAS, Leusden.

Ruhf, K.; Immerman, G; Toensmeier, E., et al. (2003), “Who will farm”, en The Natural Farmer, special supplement on beginning farmers, 55(2), pp. 1-16.

Schumacher, E.F. (1983) [1973], Lo pequeño es hermoso. Hermann Blume 
Ediciones, Madrid.

Seccombe, W. (2007), A home-grown strategy for Ontario agriculture. A new deal for farmers, a new relationship with consumers. Report prepared for the Toronto Food Policy Council.

Sevilla Guzmán, E. (1979), La evolución del campesinado en España. Península, Barcelona.

Ídem (2006), De la Sociología Rural a la Agroecologia. Icaria, Barcelona.

Tisenkopfs, T; Lace, I. i Mierina, I. (2010), “Social Capital” en Ploeg, J.D. van der i Marsden, T. (Eds.), Unfolding Webs. The dynamics of regional rural development. Assen, Països Baixos, Royal van Gorcum, pp, 87-110.

Toledo V.M.; Alarcón-Chaires P. i Barón L. (1999), “Estudiar lo rural desde una perspectiva interdisciplinaria, una aproximación al caso de México”, en Estudios Agrarios, 12, pp. 55-90.

Ventura, F.; Milone, P. i Ploeg, J.D. van der (2010), "Understanding rural development dynamics”, en Milone, P. i Ventura, F. (Eds.), Networking the rural. The future of green regions in Europe. Royal van Gorcum, Assen, Països Baixos.

Viladomiu, L. (2003), “Noves tendències de desenvolupament rural a Europa”, en Quaderns Agraris, 28, pp. 21-36.

Webb, M. (2008), Apples to Oysters. A food lover's Tour of Canadian Farms. Viking Canada, Canada.

Williams, F. i Farrington, J. (2006), "Succession and the future of farming, problem or perception?”, en The Rural citizen, governance, culture and wellbeing in the 21st century. 4-7 de abril de 2006, Plymouth.

Recibido: 18.02.2013

Aceptado: 07.03.2013 